\title{
Groups in which some primary subgroups are weakly s-supplemented*
}

\author{
Baojun Li \\ College of Applied Mathematics, Chengdu University of Information Technology \\ Chengdu 610225, P. R. China \\ E-mail: baojunli@cuit.edu.cn
}

\begin{abstract}
A subgroup $H$ of a group $G$ is called weakly s-supplemented in $G$ if there is a subgroup $T$ such that $G=H T$ and $H \cap T \leq H_{s G}$, where $H_{s G}$ is the subgroup of $H$ generated by all those subgroups of $H$ which are s-permutable in $G$. The influence of primary weakly s-supplemented subgroups on the structure of finite groups is investigated. An open question promoted by Skiba is studied and some known results are generalized.
\end{abstract}

\section{Introduction}

All groups considered in this paper are finite. The notions and notations not introduced are standard and the reader is referred to [1] 3 if necessary.

A subgroup $H$ of a group $G$ is said to be a permutable subgroup (cf. 1]) of $G$ or a quasinormal subgroup of $G$ (cf. [4) if $H$ is permutable with all subgroups of $G$. The permutability of subgroups plays an important role in the study of the structure of finite groups and was generalized extensively. Recall that a subgroup $H$ of a group $G$ is called s-permutable (or s-quasinormal) in $G$ if $H$ permutes with every Sylow subgroup of $G$ (cf. [5]). Let $H$ be a subgroup of $G$. $H_{s G}$ denotes the subgroup of $H$ generated by all those subgroups of $H$ which are s-permutable in $G$. In [6], the following definitions are introduced.

Definition 1.1 6] Let $H$ be a subgroup $G$. $H$ is called weakly s-supplemented in $G$ if there is a subgroup $T$ such that $G=H T$ and $H \cap T \leq H_{s G}$, and if $T$ is subnormal in $G$ then $H$ is called weakly s-permutable in $G$

By using this idea, Skiba 6] proved the following nice result.

* The author was supported by NNSF of P. R. China (Grant 11071229), Scientific Research Foundation of CUIT(Grant J201114) and a China Postdoctoral Science Foundation funded project (Grant 20110491726) 
Theorem 1.2 Let $\mathfrak{F}$ be a saturated formation containing all supersoluble groups and $G$ a group with a normal subgroup $E$ such that $G / E \in \mathfrak{F}$. Suppose that every non-cyclic Sylow subgroup $P$ of $E$ has a subgroup $D$ such that $1<|D|<|P|$ and all subgroups $H$ of $P$ with order $|H|=|D|$ and with order $2|D|$ (if $P$ is a non-abelian 2-group and $|P: D|>2$ ) not having a supersoluble supplement in $G$ are weakly s-permutable in $G$. Then $G \in \mathfrak{F}$.

The above theorem generalized many known results. in connection with this, the following question was proposed by A. Skiba.

Question 1.3 [6, Question 6.4] Let $\mathfrak{F}$ be a saturated formation containing all supersolvable groups and $G$ a group with a normal subgroup $E$ such that $G / E \in \mathfrak{F}$. Suppose that every non-cyclic Sylow subgroup $P$ of $E$ has a subgroup $D$ such that $1<|D|<|P|$ and all subgroups $H$ of $P$ with order $|H|=|D|$ and with order $2|D|$ (if $P$ is a non-abelian 2-group and $|P: D|>2$ ) are weakly s-supplemented in $G$. Is then $G \in \mathfrak{F}$ ?

We have given an example in [7] to show that the answer of this question is negative in general. But, in the following theorem, we will prove in many case the question has positive answer.

For convenience, if $m=p^{\alpha}$ is a $p$-number, let $\iota(m)$ denote $\log _{p} m=\alpha$ and if $P$ is a $p$ group we use $\iota(P)$ instead of $\iota(|P|)$.

Theorem 1.4 Let $\mathfrak{F}$ be a saturated formation containing all supersolvable groups and $G$ a group with a normal subgroup $E$ such that $G / E \in \mathfrak{F}$. Suppose that every non-cyclic Sylow subgroup $P$ of $E$ has a subgroup $D$ such that $1<|D|<|P|$ and all subgroups $H$ of $P$ with order $|H|=|D|$ and with order $2|D|$ (if $P$ is a non-abelian 2-group and $|P: D|>2$ ) having no supersolvable supplement in $G$ are weakly s-supplemented in $G$. If one of the following holds:

(i) $\Phi(P) \neq P^{\prime}$;

(ii) $|D| \leq\left|P^{\prime}\right|$;

(iii) $\left|P^{\prime}\right|<|D|$ and $\left(\iota\left(P / P^{\prime}\right), \iota\left(|D| /\left|P^{\prime}\right|\right)\right)=1$ or $(\iota(P), \iota(|P: D|))=1$;

then $G \in \mathfrak{F}$

\section{Elementary Properties}

Lemma 2.1 ( 6, Lemma 2.10]) Let $G$ be a group and $H \leq K \leq G$. Then

(i) Suppose that $H$ is normal in $G$. Then $K / H$ is weakly s-supplemented in $G / H$ if and only if $K$ is weakly s-supplemented in $G$.

(ii) If $H$ is weakly s-supplemented in $G$, then $H$ is weakly s-supplemented in $K$.

(iii) Suppose that $H$ is normal in $G$. Then the $H E / H$ is weakly s-supplemented in $G / H$ for every weakly weakly s-supplemented in $G$ subgroup E satisfying $(|H|,|E|)=1$.

Lemma 2.2 ( [8, Lemma 2.8]) Let $\mathfrak{F}$ be a saturated formation and $P$ be a normal $p$-subgroup of $G$. Then $P \subseteq Z_{\infty}^{\mathfrak{F}}(G)$ if and only if $P / \Phi(P) \subseteq Z_{\infty}^{\mathfrak{F}}(G / \Phi(P))$. 
Lemma 2.3 ( 6, Lemma 2.7]) If $H$ is s-permutable in a group $G$ and $H$ is a p-group for some prime $p$, then $O^{p}(G) \leq N_{G}(H)$.

Lemma 2.4 ( [3, Lemma3.8.7] Let $G$ be a p-solvable group. If $O_{p^{\prime}}(G)=1$ and $O_{p}(G) \leq H \leq G$, then $O_{p^{\prime}}(H)=1$

The following Lemma is well known.

Lemma 2.5 Let $N$ be a nilpotent normal subgroup of $G$. If $N \cap \Phi(G)=1$ then $N \leq \operatorname{Soc}(G)$, that is, $N=N_{1} \times N_{2} \times \cdots \times N_{r}$, where $N_{1}, N_{2}, \cdots, N_{r}$ are minimal normal subgroups of $G$.

Lemma 2.6 ( 9]) Let $G$ be a nonabelian simple group and $H$ a subgroup of $G$. If $|G: H|=p^{a}$, where $p$ is a prime. Then one of the following holds:

(i) $G=A_{n}, H \cong A_{n-1}$, where $n=p^{a}$;

(ii) $G=P S L_{n}(q),|G: H|=\left(q^{n}-1\right) /(q-1)=p^{a}$;

(iii) $G=P S L_{n}(11), H \cong A_{5}$;

(iv) $G=M_{23}$ and $H \cong M_{22}$ or $G=M_{11}$ and $H \cong M_{10}$;

(v) $G=\mathrm{PSU}_{4}(2)$, the index of $\mathrm{H}$ in $G$ is 27.

Lemma 2.7 Let $G$ be a group, $p$ the minimal prime divisor of the order of $G$ and $P$ a Sylow p-subgroup of $G$. If every maximal subgroup of $P$ having no supersolvable supplement in $G$ is weakly s-supplemented in $G$ then $G$ is p-nilpotent.

Proof It can be obtained directly from [7, Theorem C].

Lemma 2.8 ( [2, III, 5.2 and IV, 5.4]) Suppose that $p$ is a prime and $G$ is a minimal non-p-nilpotent group. Then

(i) $G$ has a normal Sylow p-subgroup $P$ and $G=P Q$, where $Q$ is a non-normal cyclic q-subgroup for some prime $q \neq p$.

(ii) $P / \Phi(P)$ is a minimal normal subgroup of $G / \Phi(P)$.

(iii) If $P$ is abelian or $p>2$, then $\exp (P)=p$.

(iv) If $P$ is non-abelian and $p=2$, then $\exp (P)=4$.

Lemma 2.9 Let $G$ be a group, $p$ the minimal prime divisor of the order of $G$ and $P$ a Sylow p-subgroup of $G$. If every subgroup of $P$ of order $p$ or 4(when $P$ is a non abelian 2-group) having no supersolvable supplement in $G$ is weakly s-supplemented in $G$ then $G$ is p-nilpotent.

Proof By Lemma 2.1 one can verify that the hypotheses are subgroups closed. Thus if $G$ is not $p$-nilpotent then we can assume that $G$ is a minimal non- $p$-nilpotent group, and hence, by Lemma 2.8. $G=P \rtimes Q$, where $Q$ is a cyclic $q$-group for some prime $q, P / \Phi(P)$ is a chief factor of $G$ and $\exp P=p$ or 4 (when $P$ is noncyclic 2-group). Let $a \in P \backslash \Phi(P)$ and $H=\langle a\rangle$. Then $|H|=p$ or 4 (when $P$ is a nonabelian 2-group). Thus $H$ either has a supersolvable supplement in $G$ or is weakly s-supplemented in $G$. Assume $|H|=2$. If $H$ has a complement $T$ in $G$, then $T$ is $p$-nilpotent since $G$ is minimal non-p-nilpotent group. Since $|G: T|=|H T: T|=|H|=p, T \unlhd G$. This induces that $G$ is $p$-nilpotent, a contradiction. Thus $G$ 
is the only supplement of $H$ in $G$. If $H$ has a supersolvable supplement in $G$, then $G$ is supersolvable and so is $p$-nilpotent since $p$ is minimal. If $H$ is weakly s-supplemented in $G$ then $H$ is s-permutable in $G$ and hence $H \Phi(P) / \Phi(P)$ is s-permutable in $G / \Phi(P)$. It follows from Lemma 2.3 that $H \Phi(P) / \Phi(P) \unlhd G / \Phi(P)$ and so $P / \Phi(P)=H \Phi(P)$ is cyclic of order $p$. Hence $|G: Q \Phi(P)|=p$ and so $Q \phi(P) \unlhd G$. This implies that $G / \Phi(P)$ is cyclic and so is $G$, a contradiction. If $|H|=4$, considering the subgroup $H \Phi(P) / \Phi(P)$ in $G / \Phi(P)$, a contradiction can also be obtained by a similar argument. Therefore the lemma holds.

\section{Proof of Theorem 1.4}

Lemma 3.1 Let $P$ be a normal p-subgroup of $G$ with $P \cap \Phi(G)=1$. Assume that $D$ is a subgroup of $P$ with $1<D<P$. If every subgroup of order $|D|$ of $P$ having no supersolvable supplement in $G$ is weakly s-supplement in $G$, then $P=P_{1} \times P_{2} \times \cdots \times P_{r}$, where $P_{1}, P_{2}, \cdots, P_{r}$ are all minimal normal in $G$ of same order and $\iota(D)=m \iota\left(P_{i}\right)$, that $i s,|D|=\left|P_{i}\right|^{m}$ for some positive integer $m, i=1,2, \cdots, r$.

Proof By Lemma 2.5, $P=P_{1} \times P_{2} \times \cdots \times P_{r}$, where $P_{1}, P_{2}, \cdots, P_{r}$ are all minimal normal in $G$. Assume that there is a $P_{i}$ such that $|D|<\left|P_{i}\right|$. Then $P_{i}$ has a proper subgroup $H$ of order $|D|$. Moreover, by the property of $p$-groups we can choose $H$ to be normal in some Sylow $p$-subgroup $G_{p}$ of $G$ containing $P$. By the hypotheses, $H$ either has a supersolvable supplement in $G$ or is weakly s-supplement in $G$. Let $T$ be a supplement of $H$ in $G$. Then $G=H T=P_{i} T$ and $P_{i} \cap T \neq 1$ since $H$ is proper in $P_{i}$. As $P_{i}$ is abelian, $P_{i} \cap T \unlhd P_{i} T=G$. But $P_{i}$ is minimal normal in $G$, so $P_{i} \cap T=P_{i}$ and thereby $T=G$. Thus $G$ is the only supplement of $H$ in $G$. If $H$ has a supersolvable supplement in $G$, then $G$ is supersolvable and $\left|P_{i}\right|=p$, which contradicts that $|D|<\left|P_{i}\right|$. If $H$ is weakly s-supplement in $G$ then $H=H \cap G$ is s-permutable in $G$. By Lemma 2.3 $O^{p}(G) \leq N_{G}(H)$. It follows that $H \unlhd G=G_{p} O^{p}(G)$. This is nonsense for $P_{i}$ is minimal normal in $G$. Thus $|D| \geq\left|P_{i}\right|$ for any $i$.

If $\left|P_{i}\right|=|D|$ for any $i$, then we can see that the conclusion holds. Assume $\left|P_{i}\right|<|D|$ for some $i$. Without loss of generality, we can assume that $i=1$. Clearly $P / P_{1}=P_{2} P_{1} / P_{1} \times \cdots \times P_{r} P_{1} / P_{1}$ and $P / P_{1} \cap \Phi\left(G / P_{1}\right)=1$ by [1, A,(9.11)]. By Lemma 2.1 one can verify that the hypotheses still hold on $G / P_{1}$. Thus $\left|P_{2} P_{1} / P_{1}\right|=\cdots=\left|P_{r} P_{1} / P_{1}\right|$ and $|D| /\left|P_{1}\right|=\left|P_{2} P_{1} / P_{1}\right|^{m_{1}}$ for some positive integer $m_{1}$. It follows that $\left|P_{2}\right|=\cdots=\left|P_{r}\right|$ and $|D| /\left|P_{1}\right|=\left|P_{2}\right|^{m_{1}}$. In particularly, $\left|P_{2}\right|<|D|$ and the hypotheses also hold on $G / P_{2}$. If $r \geq 3$, then $\left|P_{1}\right|=\left|P_{3}\right|=\cdots=\left|P_{r}\right|$. It follows that $\left|P_{1}\right|=\left|P_{2}\right|=\left|P_{3}\right|=\cdots=\left|P_{r}\right|$ and $|D|=\left|P_{1}\right|^{m_{1}+1}=\left|P_{1}\right|^{m}$ where $m=m_{1}+1$. If $r=2$ then $P / P_{1} \cong P_{1} P_{2} / P_{1}$ is minimal normal in $G / P_{1}$. Since the hypotheses holds on $G / P_{1}$, we can find a contradiction as above. Thus the lemma holds.

Corollary 3.2 Let $P$ be a normal $p$-subgroup of $G$ with $P \cap \Phi(G)=1$. Assume that $D$ is a subgroup of $P$ with $1<D<P$ and every subgroup of order $|D|$ of $P$ having no supersolvable supplement in $G$ is weakly s-supplement in $G$. If $(\iota(P), \iota(D))=1$ or $(\iota(|P: D|), \iota(D))=1$ then $P \subseteq Z_{\infty}^{\mathfrak{U}}(G)$.

Proof By Lemma 3.1 $P=P_{1} \times P_{2} \times \cdots \times P_{r}$, where $P_{1}, P_{2}, \cdots, P_{r}$ are all minimal normal in $G$, and $\iota\left(P_{i}\right), i=1, \cdots, r$, is a common divisor of $\iota(P), \iota(D)$ and $\iota(|P: D|)$. Thus if $(\iota(P), \iota(D))=1$ or $(\iota(|P: D|), \iota(D))=1$ then $P \subseteq Z_{\infty}^{\mathfrak{U}}(G)$. 
Lemma 3.3 Let $P$ be a normal p-subgroup of $G$ and $D$ a subgroup of $P$ with $1<D<P$. Assume that every subgroup of order $|D|$ or $2|D|$ (when $P$ is a nonabelian 2-group) of $P$ having no supersolvable supplement in $G$ is weakly s-supplement in $G$. If $P^{\prime}<P \cap \Phi(G)$ or $|D| \leq\left|P^{\prime}\right|$, then $P \subseteq Z_{\infty}^{\mathfrak{U}}(G)$.

Proof Assume that the lemma does not hold and choose $P$ be a counter example with minimal order. We prove the lemma via the following steps.

(1) $P \nsubseteq \Phi(G)$ and $P / P \cap \Phi(G)=P_{1} / P \cap \Phi(G) \times P_{2} / P \cap \Phi(G) \times \cdots P_{r} / P \cap \Phi(G)$, where $P_{i} / P \cap \Phi(G), i=$ $1,2, \cdots, r$, is minimal normal in $G / P \cap \Phi(G)$.

If $P \subseteq \Phi(G)$, then for any subgroup $H$ of $P, G$ is the only supplement of $H$ in $G$. If there is a subgroup $H$ of order $|D|$ (or $2|D|$ when $P$ is an nonabelian 2-group) has a supersolvable supplement in $G$ then $G$ is supersolvable and $P \subseteq Z_{\infty}^{\mathfrak{U}}(G)$. Now assume that every such subgroup $H$ has no supersolvable supplement in $G$. Then $H$ is weakly s-supplement in $G$ and hence $H=H \cap G$ is s-permutable in $G$. It follows from [10, Lemma 3.1] that $P \subseteq Z_{\infty}^{\mathfrak{U}}(G)$. This contradiction shows that $P \nsubseteq \Phi(G)$. Clearly, $(P / P \cap \Phi(G)) \cap$ $\Phi(G / P \cap \Phi(G))=1$. Hence by Lemma 2.5. $P / P \cap \Phi(G)=P_{1} / P \cap \Phi(G) \times P_{2} / P \cap \Phi(G) \times \cdots P_{r} / P \cap \Phi(G)$, where $P_{i} / P \cap \Phi(G), i=1,2, \cdots, r$, is minimal normal in $G / P \cap \Phi(G)$.

(2) $|D|>p$.

Assume that $|D|=p$. Then the hypotheses hold on $P_{i}$ for any $i \in\{1, \cdots, r\}$. If $r>1$, then $P_{i} \subseteq Z_{\infty}^{\mathfrak{U}}(G)$ for any $i$ and so $P \subseteq Z_{\infty}^{\mathfrak{U}}(G)$. Assume that $r=1$. Then $P / P \cap \Phi(G)$ is a $G$-chief factor. If $P \cap \Phi(G)=p$, then $P \cap \Phi(G) \subseteq Z_{\infty}^{\mathfrak{U}}(G)$ clearly holds. If $P \cap \Phi(G) \neq 1$ then the hypotheses hold on $P \cap \Phi(G)$ and hence $P \cap \Phi(G) \subseteq Z_{\infty}^{\mathfrak{U}}(G)$. Thus $P \cap \Phi(G) \subseteq Z_{\infty}^{\mathfrak{U}}(G)$ always holds. Let $H$ be any cyclic subgroup of order $p$ or 4 (if $P$ is a nonabelian 2-group). If $H(P \cap \Phi(G))=P$ then $P / P \cap \Phi(G) \cong H / H \cap \Phi(G)$ is cyclic. Since $P \cap \Phi(G) \subseteq Z_{\infty}^{\mathfrak{U}}(G)$, we see that $P \subseteq Z_{\infty}^{\mathfrak{U}}(G)$. Assume that $H(P \cap \Phi(G))<P$ for any such subgroup $H$. Let $T$ be any supplement of $H$ in $G$. We claim that $T=G$. If $T<G$, then $T(P \cap \Phi(G))<G$. Since $P T=H T=G$ and $P / P \cap \Phi(G)$ is an abelian minimal normal subgroup of $G / P \cap \Phi(G), T(P \cap \Phi(G)) / P \cap \Phi(G)$ is a complement of $P / P \cap \Phi(G)$. But $G /(P \cap \Phi(G))=H T /(P \cap \Phi(G))$, so $P=H(P \cap \Phi(G))$, a contradiction. Thus our claim holds. Consequently, $G$ is the only supplement of $H$ in $G$. Hence $H$ is s-permutable in $G$ if $H$ has no supersolvable supplement in $G$ by the hypotheses. By [10. Lemma 3.1], $P \subseteq Z_{\infty}^{\mathfrak{U}}(G)$, a contradiction. Hence (2) holds.

(3) $\left|P^{\prime}\right|<|D|$.

Assume that $|D| \leq\left|P^{\prime}\right|$. Since $P^{\prime} \leq P \cap \Phi(G)<P_{i},|D|<\left|P_{i}\right|, i=1,2, \cdots, r$. To prove $P \subseteq Z_{\infty}^{\mathfrak{U}}(G)$, it is sufficient to prove that $P_{i} \subseteq Z_{\infty}^{\mathfrak{U}}(G)$ for every $i$. If $P_{i}^{\prime}=P^{\prime}$, then $|D|<\left|P_{i}^{\prime}\right|$. If $P_{i}^{\prime}<P^{\prime}$, then $P_{i}^{\prime}<P_{i} \cap \Phi(G)$ since $P^{\prime} \leq \Phi(P) \leq \Phi(G)$. Therefore, the hypotheses still hold on $\left(G, P_{i}\right)$. If $P_{i}<P$, then by induction on $|P|$, we have that $P_{i} \subseteq Z_{\infty}^{\mathfrak{U}}(G)$. Now, assume that $P_{i}=P$. Then $P / P \cap \Phi(G)$ is a chief factor of $G$.

Suppose that $P^{\prime}=P \cap \Phi(G)$. Then $P / P^{\prime}$ is a chief factor of $G$. Let $H$ be a subgroup of order $|D|$ (or $2|D|$ when $P$ is an nonabelian 2-group) of $P$. We claim that $G$ is the only supplement of $H$ in $G$. Clearly, if $H \leq P^{\prime} \leq \Phi(G)$, then $H T=G$ if and only if $T=G$. Assume that $H \nsubseteq P^{\prime}$ and $T$ is a supplement of $H$ in $G$. If $T<G$, then $T P^{\prime}$ is still a proper subgroup of $G$. Since $G=H T=P T$, we have that 
$\left(P / P^{\prime}\right)\left(T P^{\prime} / P^{\prime}\right)=G / P^{\prime}$. As $P / P^{\prime}$ is minimal normal in $G / P \cap \Phi(G)$ and $T P^{\prime} / P^{\prime}$ is proper in $G / P^{\prime}$, $\left(P / P^{\prime}\right) \cap\left(T P^{\prime} / P^{\prime}\right)=1$ and $T P^{\prime} / P^{\prime}$ is a complement of $P / P^{\prime}$ in $G / P^{\prime}$. But $G=H T$, so $T P^{\prime} / P^{\prime}$ is a supplement of $H P^{\prime} / P^{\prime}$ in $G / P^{\prime}$. This induces that $H P^{\prime} / P^{\prime}=P / P^{\prime}$ and so $H=P$, a contradiction. Thus our claim holds. It follows that all subgroups of order $|D|$ (and $2|D|$ when $P$ is an nonabelian 2-group) in $P$ having no supersolvable supplement in $G$ are s-permutable in $G$ and hence $P \subseteq Z_{\infty}^{\mathfrak{U}}(G)$ by [10, Lemma $3.1]$.

Assume that $P^{\prime}<P \cap \Phi(G)$. Then by induction on $P, P \cap \Phi(G) \subseteq Z_{\infty}^{\mathfrak{U}}(G)$. Let $N$ be a minimal normal subgroup of $G$ contained in $P^{\prime}<P \cap \Phi(G)$. Then $N$ is of order $p$. By (2), $|D|>p$ and so the hypotheses still hold on $(G / N, P / N)$ by Lemma 2.1. Hence $P / N \subseteq Z_{\infty}^{\mathfrak{U}}(G / N)$. It follows directly from $|N|=p$ that $P \subseteq Z_{\infty}^{\mathfrak{U}}(G)$. This contradicts the choice of $P$ and hence $\left|P^{\prime}\right|<|D|$.

(4) $P^{\prime}=1$ and $P$ is abelian.

Since $|D| \nless\left|P^{\prime}\right|$ by $(3), P^{\prime}<P \cap \Phi(G)$ by the hypotheses. Then it can be verified that the hypotheses hold on $\left(G / P^{\prime}, P / P^{\prime}\right)$ by Lemma 2.1. If $P^{\prime} \neq 1$, then $P / P^{\prime} \subseteq Z_{\infty}^{\mathfrak{U}}\left(G / P^{\prime}\right)$ by induction. Since $P^{\prime} \leq \Phi(P)$, It follows from Lemma 2.2 that $P \subseteq Z_{\infty}^{\mathfrak{U}}(G)$, which contradicts the choice of $P$. Thus (4) holds.

(5) Let $N$ be a minimal normal subgroup of $G$ contained in $P \cap \Phi(G)$. Then $|N|<|D|$

If $|D|<|N|$, then the hypotheses holds on $(G, N)$ and hence $N \subseteq Z_{\infty}^{\mathfrak{H}}(G)$ by (1). It follows that $|N|=p$ and $|N| \leq|D|$, a contradiction.

Suppose that $|N|=|D|$. In this case, we claim that $N$ is cyclic. Let $L / N$ be a chief factor of $G_{p}$, where $G_{p}$ is a Sylow $p$-subgroup of $G$. Then $|L|=p|N|=p|D|$. Let $\mho=\left\langle x^{p} \mid x \in l\right\rangle$. Then $\mho \leq \Phi(L) \leq N$. If $\mho=N$, then $L$ is cyclic since $L / \Phi(L)=L / N$ is cyclic. It follows that $N$ is cyclic. Assume that $\mho<N$. Clearly, $\mho \unlhd G_{p}$ and hence there is a maximal subgroup $M$ of $N$ such that $\mho \leq M$ and $M \unlhd G_{p}$. Choose an element $x \in L \backslash N$. Then $x^{p} \in \mho \leq M$ and $H=\langle M, x\rangle$ is of order $p|M|=|N|=|D|$. Let $T$ be any supplement of $H$ in $G$. Since $P$ is abelian, $N \cap T \unlhd P T=H T=G$. If $N \nsubseteq T$ then $N \cap T=1$ by the minimality of $N$. Thus $|N T|=|N||T| \geq|H T|=G$ and thereby, $G=N T$, which is contrary to $N \subseteq \Phi(G)$. Hence $N \subseteq T$. Assume that $H$ has a Supersolvable supplement $T$ in $G$. Then there is a cyclic subgroup $R$ of $N$ such that $R \unlhd T$. Since $P$ is abelian, $R \unlhd P T=H T=G$. By the minimality of $N$, we have that $N=R$ is cyclic. Assume that $H$ has no Supersolvable supplement in $G$. Then $H$ is weakly s-supplement in $G$ by hypotheses. It follows that $M=H \cap T \cap N$ is s-permutable in $G$. Since $M \unlhd G_{p}, M \unlhd G=G_{p} O^{p}(G)$. Again by the minimality of $N$, we have that $M=1$ and so $N$ is cyclic. Thus our claim holds. This implies that $|N|=|D|=p$. But $|D|>p$ by (2), a contradiction. Hence $|N|<|D|$ and (5) holds.

By the hypotheses, we can see that $P \cap \Phi(G) \neq 1$. In the following, $N$ denotes always a minimal normal subgroup of $G$ contained in $P \cap \Phi(G)$.

(6) $N$ is the unique minimal normal subgroup of $G$ contained in $P$.

Assume that this does not holds and $P$ contains a minimal normal subgroup $L$ of $G$ different from $N$. Since $|N|<|D|$ by (5), by Lemma 2.1 every subgroup of order $|D| /|N|$ of $P / N$ having no supersolvable supplement in $G / N$ is weakly s-supplemented in $G / N$. If $P / N \cap \Phi(G / N) \neq 1$ then the hypotheses still hold on $(G / N, P / N)$ and so $P / N \subseteq Z_{\infty}^{\mathfrak{U}}(G / N)$. Hence $L$ is of order $p$ and $|L|<|D|$ since $|D|>p$ by (2). If $P / N \cap \Phi(G / N)=1$, then by Lemma $3.1 P / N=P_{1} / N \times P_{2} / N \times \cdots \times P_{r} / N$, where $P_{1} / N, P_{2} / N, \cdots, P_{r} / N$ 
are all minimal normal in $G / N,\left|P_{1} / N\right|=\left|P_{2} / N\right|=\cdots=\left|P_{r} / N\right|$ and $|D| /|N|=\left|P_{1} / N\right|^{m}$ for some positive integer $m$. In particularly, $|L|=\left|P_{1} / N\right|<|D|$. Thus $|L|<|D|$ holds in both case and hence the hypotheses hold on $(G / L, P / L)$ since, clearly, $N L / L$ is contained in $\Phi(G / L)$. Thus $P / L \subseteq Z_{\infty}^{\mathfrak{U}}(G / L)$. In particularly, $N \cong N L / L$ is cyclic. If $P / N \subseteq Z_{\infty}^{\mathfrak{U}}(G / N)$, then $P \subseteq Z_{\infty}^{\mathfrak{U}}(G)$. Thus $P / N \cap \Phi(G / N)=1$. Also, if $L$ is cyclic, then $P \subseteq Z_{\infty}^{\mathfrak{U}}(G)$ since $P / L \subseteq Z_{\infty}^{\mathfrak{U}}(G / L)$. Assume that $L$ is noncyclic and $P / N=P_{1} / N \times P_{2} / N \times \cdots \times P_{r} / N$, where $P_{1} / N, P_{2} / N, \cdots, P_{r} / N$ are all minimal normal in $G / N$ and $\left|P_{1} / N\right|=\left|P_{2} / N\right|=\cdots=\left|P_{r} / N\right|$. Since $P / L \subseteq Z_{\infty}^{\mathfrak{U}}(G / L)$, any $G$-chief factor between $L$ and $P$ is cyclic. Thus if $r \neq 1$, then by [3, Theorem 1.6.8], there is some $i$ such that $P_{i} / N \cong L$ is noncyclic and $P_{j} / N \cong Q / L$ is cyclic for any $j \neq i$, where $Q / L$ is a $G$-chief factor contained in $P$. But $\left|P_{i} / N\right|=\left|P_{j} / N\right|$, a contradiction. Hence $r=1$. This induces that $P / N$ is minimal normal in $G / N$ and $|P / N|=\left|P_{1} / N\right|<|D| /|N|$, a contradiction. Thus (6) holds.

(7) $\Phi(P)=1$.

Assume that $\Phi(P) \neq 1$. Then $N \subseteq \Phi(P)$ by (6). If $P / N \subseteq Z_{\infty}^{\mathfrak{U}}(G / N)$, then $P \subseteq Z_{\infty}^{\mathfrak{U}}(G)$ by Lemma 2.2 Suppose that $P / N \nsubseteq Z_{\infty}^{\mathfrak{U}}(G / N)$. Then $\Phi(G / N) \cap P / N=1$ and $P / N=P_{1} / N \times P_{2} / N \times \cdots \times P_{r} / N$ by Lemma 3.1. Moreover, $\left|P_{1} / N\right|=\left|P_{2} / N\right|=\cdots=\left|P_{r} / N\right|$ and $|D| /|N|=\left|P_{1} / N\right|^{m}$ for some positive integer $m$. Assume that $\exp P=p$. Then $\Phi(P)=\mho_{1}(P)=1$, where $\mho_{1}(P)=\left\{a^{p} \mid a \in P\right\}<P$. This contradicts $\Phi(P) \neq 1$. Hence $\exp P>p^{2}$. Then $\exp (P / N)>p$ and so $\Phi(P / N) \neq 1$. This contradicts that $\Phi(G / N) \cap P / N=1$. Thus $\exp P=p^{2}$. If $D$ is maximal in $P$, then $p=|P: D|=|P / N: D / N|=\frac{\left|P_{1} / N\right|^{r}}{\left|P_{1} / N\right|^{m}}$. Hence $r=m+1$ and $\left|P_{1} / N\right|=\left|P_{2} / N\right|=\cdots=\left|P_{r} / N\right|=p$. This induces that $P / N \subseteq Z_{\infty}^{\mathfrak{U}}(G / N)$, a contradiction. Thus $|P: D| \geq p^{2}$. We claim that $N$ is cyclic. Otherwise, there must be a subgroup $H$ of order $|D|$ such that $H \cap N \neq 1$ since $\exp P=p^{2}$ and $|P: D| \geq p^{2}$. Moreover, we can choose that $H \cap N \unlhd G_{p}$, where $G_{p}$ is some Sylow $p$-subgroup of $G$. Let $T$ be any supplement of $H$ in $G$. Then $P \cap T \unlhd P T=H T=G$. Clearly $P \cap T \neq 1$ so $N \subseteq T$ as $N$ is the only minimal normal subgroup of $G$ contained in $P$. If $T$ is supersolvable, then $N$ has a cyclic subgroup $R$, which is normal in $T$. But $P$ is abelian, so $R$ is normal in $G$ and hence $N=R$ is cyclic. Assume that $H$ is weakly s-supplemented in G. Then $N \cap H \leq T \cap H \leq H_{s G}$ and so $H \cap N=H_{s G} \cap N$ is s-permutable in $G$. This induces that $O^{p}(G) \subseteq N_{G}(N \cap H)$ and so $N \cap H \unlhd G$, which contradicts the minimality of $N$. Thus $N$ is cyclic and our claim holds.

Since $\Phi((G / N) \cap(P / N))=1$, we have that $N=\Phi(P)$. Let $P=\left\langle a_{1}\right\rangle \times \cdots\left\langle a_{k}\right\rangle \times\left\langle a_{k+1}\right\rangle \times \cdots \times\left\langle a_{n}\right\rangle$, where $\left|a_{1}\right|=\cdots=\left|a_{k}\right|=p^{2}$ and $\left|a_{k+1}\right|=\cdots=\left|a_{n}\right|=p$. Then $k=1$ since $N=\Phi(P)$ is of order $p$. It follows that $\Omega=\Omega_{1}(P)=\left\{a \mid a^{p}=1\right\}$ is a maximal subgroup of $P$. Clearly, $\Omega \cap \Phi(G)=P \cap \Phi(G) \neq 1$. Note that $D$ is not maximal in $P$. Hence $|D|<|\Omega|$ and so $\Omega \subseteq Z_{\infty}^{\mathfrak{U}}(G)$ by induction. But $P / \Omega$ is of order $p$, so $P \subseteq Z_{\infty}^{\mathfrak{U}}(G)$. This contradiction shows that (7) holds.

The final contradiction

Since $\Phi(P)=1, P$ is an elementary abelian $p$-group. In particularly, $N$ is complemented in $P$. Assume that $N$ is noncyclic. Then there exists a subgroup $H$ of order $|D|$ such that $1<H \cap N<N$ and $H \cap N \unlhd G_{p}$, where $G_{p}$ is a Sylow $p$-subgroup of $G$. As above argument, one can find a contradiction. Thus $N$ is cyclic. Let $H_{1} / N$ be any subgroup of $P / N$ of order $|D|$ and $H$ be a complement of $N$ in $H_{1}$. Then $H$ is of order $|D|$. If $H_{1} / N$ has no supersolvable supplement in $G / N$, then $H$ has no supersolvable 
supplement in $G$ and so is weakly s-supplement in $G$ by hypotheses. Let $T$ be any supplement of $H$ in $G$ with $H \cap T \leq H_{s G}$. Since $N \leq T$ by above argument, $T / N$ is a supplement of $H_{1} / N$ in $G / N$ and $\left(H_{1} / N\right) \cap(T / N)=\left(H_{1} \cap T\right) / N=(H \cap T) N / N \leq H_{s G} N / N$. Hence $H_{1} / N$ is weakly s-supplemented in $G / N$. If $P / N \cap \Phi(G / N) \neq 1$, then the hypotheses hold on $(G / N, P / N)$ and so $P / N \subseteq Z_{\infty}^{\mathfrak{U}}(G / N)$. It follows from $|N|=p$ that $P \subseteq Z_{\infty}^{\mathfrak{U}}(G)$. If $P / N \cap \Phi(G / N)=1$, then $P / N=Q_{1} / N \times Q_{2} / N \times \cdots \times Q_{s} / N$, where $Q_{i} / N$ is minimal normal in $G, i=1, \cdots, s,\left|Q_{1} / N\right|=\left|Q_{2} / N\right|=\cdots=\left|Q_{s} / N\right|$ and $|D|=\left|Q_{1} / N\right|^{m^{\prime}}$ for some integer $m^{\prime}$ by Lemma 3.1. But we have that $P / N=P_{1} / N \times P_{2} / N \times \cdots \times P_{r} / N$, so $\left|Q_{1} / N\right|=\left|P_{1} / N\right|$ by [3, Theorem 1.6.8]. Thus $\left|P_{1} / N\right|^{m}=|D| / p=\left|P_{1} / N\right|^{m^{\prime}} / p$ for some integers $m$ and $m^{\prime}$. This implies that $m^{\prime}=m+1$ and $\left|P_{1} / N\right|=p$. Therefore $P / N \subseteq Z_{\infty}^{\mathfrak{U}}(G / N)$ and then, $P \subseteq Z_{\infty}^{\mathfrak{U}}(G)$ is cyclic since $N$ is cyclic. The final contradiction completes the proof.

Lemma 3.4 Let $G$ be a group and $p$ the minimal prime divisor of the order of $G$. Assume that $P$ is a Sylow p-subgroup of $G$ and $D$ is a nontrivial proper subgroup of $P$. If every subgroup of $P$ of order $|D|$ or $2|D|$ (when $P$ is a nonabelian 2-group) having no supersolvable supplement in $G$ is weakly s-supplemented in $G$, then $G$ is p-solvable and the p-length of $G$ is 1 .

Proof Assume the lemma does not holds and let $G$ be a counter example of minimal order. Then $G$ is not $p$-nilpotent. We proceed the proof via the following steps.

(1) $O_{p^{\prime}}(G)=1$

By Lemma 2.1 it can be verified that the hypotheses still hold on $G / O_{p^{\prime}}(G)$ and if $O_{p^{\prime}}(G) \neq 1$ then $G / O_{p^{\prime}}(G)$ is $p$-solvable and the $p$-length is 1 . It follows that $G$ is $p$-solvable and the $p$-length of $G$ is 1 . So we can assume that $O_{p^{\prime}}(G)=1$.

(2) Let $N$ be a minimal normal subgroup of $G$. If $N$ is a p-group, then $|N|=|D|$.

If $|D|<|N|$, then $N$ has a proper subgroup $H$ of order $|D|$. Since $N$ is minimal normal in $G$ and $N$ is abelian, $G$ is the only supplement of $H$ in $G$. If $H$ has a supersolvable supplement in $G$, then $G$ is supersolvable and so the $p$-length of $G 1$, which contradicts the choice of $G$. Assume that every such subgroup $H$ is weakly s-supplemented in $G$. Then $H$ is s-permutable in $G$. Without loss of generality, we can assume that $H$ is normal in $P$. Then $H \unlhd\left\langle P, O^{p}(G)\right\rangle=G$, which contradicts the minimality of $N$. Thus $|N| \leq|D|$.

If $|N|<|D|$, then the hypotheses still hold on $G / N$. Therefore, $G / N$ is $p$-solvable and the $p$-length is 1. It follows that $G$ is $p$-solvable. Assume that $G$ has another minimal normal subgroup $L$. Then, similarly, $G / L$ is also $p$-solvable and its $p$-length is 1 . This induces that $G \cong G / N \cap L$ is $p$-solvable and the $p$-length of it is 1 , a contradiction. Thus $N$ is the unique minimal normal subgroup of $G$. Since the class of all $p$-solvable groups with the $p$-length is 1 is a saturated formation, we have that $N \nsubseteq \Phi(G)$. It follows that $G=N \rtimes M$ for some maximal subgroup $M$ of $G$ and $N=O_{p}(G)$. Clearly, $O_{p^{\prime}}(M) \neq 1$ and $|P \cap M|>|D| /|N|$. Let $P_{1}$ be an subgroup of $P \cap M$ of order $p|D| /|N|$ and $M_{1}=P_{1} O_{p^{\prime}}(M) N$. Then $P_{1} N$ is a Sylow $p$-subgroup of $M_{1}$, and every maximal subgroup $H$ of $P_{1} N$ is of order $|D|$. Thus, if $H$ has no supersolvable supplement in $M_{1}$, then $H$ is weakly s-supplemented in $M_{1}$ by Lemma 2.1, and then by Lemma 2.7. $M_{1}$ is $p$-nilpotent. But $O_{p}(G)=N \leq M_{1}$, so $O_{p^{\prime}}\left(M_{1}\right)=1$ by Lemma 2.4. Thus $M_{1}$ is a 
p-group. This contradiction shows that (2) holds.

(3) $O_{p}(G)=1$

Assume that $O_{p}(G) \neq 1$. Suppose $\Phi(G) \cap O_{p}(G) \neq 1$ and let $N$ be a minimal normal subgroup of $G$ contained in $\Phi(G) \cap O_{p}(G)$. By $(2),|N|=|D|$. If $N<O_{p}(G)$ then $O_{p}(G) \subseteq Z_{\infty}^{\mathfrak{U}}(G)$ by Lemma 3.3 It follows that $O_{p}(G) \subseteq Z_{\infty}(G)$ since $p$ is the minimal prime divisor of $|G|$. Hence $N$ is cyclic and $|N|=|D|=p$. It follows from Lemma 2.9 that $G$ is $p$-nilpotent, a contradiction. Assume $N=O_{p}(G)$. Let $L / N$ be a minimal normal subgroup of $P / N$. Then $|L|=p|N|=p|D|$ and $N$ is maximal in $L$. It follows that $\mho_{1}(L)=\left\langle x^{p} \mid x \in L\right\rangle \leq \Phi(L) \leq N$. If $\mho_{1}(L)=N$, then $N=\Phi(L)$ and hence $L$ is cyclic. This induces that $N$ is cyclic and so $|D|=|N|=p$. In this case, by Lemma 2.9, $G$ is $p$-nilpotent, a contradiction. Therefore, $\mho_{1}(L)<N$. Let $M$ be a maximal subgroup of $N$ such that $M \unlhd P$ and $\mho_{1}(L) \leq M$. Choose $x \in L \backslash N$. Then $H=\langle M, x\rangle$ is a subgroup of order $|N|=|D|$. Let $T$ be a supplement of $H$ in $G$. Suppose $T<G$. Since $N \leq \Phi(G), T N<G$. But $|G: T N|=|T L: T N|=p \frac{|T \cap N|}{|T \cap L|} \leq p$, so $T N$ is maximal in $G$ and $|G: N T|=p$. Thus $N T$ is normal in $G$ by the minimality of $p$. If $N$ is not a Sylow $p$ subgroup of $N T$, then the hypotheses still hold on $N T$ and hence $N T$ is $p$-nilpotent. Since $|G: N T|=p$ and $N T \unlhd G, G$ is $p$-nilpotent. If $N$ is a Sylow $p$ subgroup of $N T$, then $D$ is maximal in $P$ and so $G$ is $p$-nilpotent by Lemma 2.7 This contradiction shows that $G$ is the only supplement of $H$ in $G$. If $T$ is supersolvable, then $G=T$ is $p$-nilpotent, a contradiction. Assume that $H$ has no supersolvable supplement in $G$. Then $H$ is weakly s-supplemented in $G$ by the hypotheses and consequently, $H=H \cap G$ is s-permutable in $G$. It follows that $M=H \cap N$ is s-permutable in $G$ and so $M \unlhd P O^{p}(G)=G$. By the minimality of $N$, we have that $M=1$ and $N$ is cyclic of order $p$. Still by Lemma 2.9, $G$ is $p$-nilpotent, a contradiction.

Suppose that $\Phi(G) \cap O_{p}(G)=1$. Then $O_{p}(G)$ is abelian. Let $N$ be a minimal normal subgroup of $G$ contained in $O_{p}(G)$. Then $N$ is of order $|D|$ by (2) and is complemented in $G$ by $\Phi(G) \cap O_{p}(G)=1$. Let $G=N \rtimes M$. Clearly, $O_{p}(M) \subseteq O_{p}(G)$. If $O_{p}(M) \neq 1$, then $O_{p}(M) \unlhd M O_{p}(G)=G$. Let $L$ be a minimal normal subgroup of $G$ contained in $O_{p}(M)$. Then the order of $L$ is $|D|$ by (2). If $L$ is a Sylow $p$-subgroup of $M$, then $O_{p}(G)=N L$ is a Sylow $p$-subgroup of $G$. This is contrary to the choice of $G$. Thus the order of a Sylow $p$-subgroup of $M$ is greater than $|D|$ and so, the hypotheses hold on $M$. Therefore, $G / N \cong M$ is $p$-solvable and the $p$-length of it is 1 . By the same argument, we have that $G / L$ is also $p$-solvable and the $p$-length of $G / L$ is 1 . Therefore, $G \cong G / L \cap N$ is $p$-solvable and the $p$-length of it is 1 , which contradicts the choice of $G$. Hence $O_{p}(M)=1$. Now assume that $O_{p^{\prime}}(M) \neq 1$. Let $x \in P \cap M$ of order $p$ and $P_{1}=\langle N, x\rangle$. Then $\left|P_{1}\right|=p|D|$. Since $N O_{p^{\prime}}(M) \unlhd G, X=O_{p^{\prime}}(M) P_{1}=O_{p^{\prime}}(G) N P_{1}$ is a subgroup of $G$ and every maximal subgroup of $P_{1}$ is of order $|D|$. Hence by Lemma 2.7 that $X$ is $p$-nilpotent and so is $N O_{p^{\prime}}(M)$. This induces that $O_{p^{\prime}}(M)$ char $N O_{p^{\prime}}(M) \unlhd G$, which contradicts $O_{p^{\prime}}(G)=1$. Thus $O_{p^{\prime}}(M)=O_{p}(M)=1$ and in particularly, $G$ is not solvable. If $p>2$, then $G$ is of odd order and so is solvable, a contradiction. Hence $p=2$. Let $R$ be a minimal subnormal subgroup of $M$. Then $R$ is nonabelian and $p=2$ is a divisor of $|R|$. Let $G_{1}=N R$. Then the hypotheses still hold on $G_{1}$ since $|N|=|D|<\left|G_{1_{p}}\right|$, where $G_{1_{p}}$ is a Sylow $p$-subgroup of $G_{1}$. If $G_{1}<G$, then $G_{1}$ is $p$-solvable and so is $R$, a contradiction. Hence $G=G_{1}=N R$. If the Sylow $p$-subgroup $P$ is abelian, then $P \cap R \unlhd P$ and so $(P \cap R)^{G}=(P \cap R)^{R} \leq R$. Since $R$ is simple, we have that $R=(P \cap R)^{G} \unlhd G$ and so $G=N \times R$. But $N$ is minimal normal in $G$, so $N$ is of order $p$. Thus 
$G$ is $p$-nilpotent by Lemma 2.9 and $|N|=|D|$. Assume that $P$ is nonabelian. Then every subgroup $H$ of order $2|D|=2|N|$ having no supersolvable supplement in $G$ is weakly s-supplemented in $G$. By Lemma 2.1 it can be verified that every subgroup of order 2 of $G / N$ having no supersolvable supplement in $G / N$ is weakly s-supplemented in $G / N$. Since $R \cong R N / N=G / N$, every subgroup of order 2 of $R$ having no supersolvable supplement in $R$ is weakly s-supplemented in $R$. Let $H$ be a subgroup of $R$ of order 2 and $T$ a supplement of $H$ in $R$. If $T \neq R$, then $|R: T|=2$ and so $T \unlhd R$, which contradicts that $R$ is simple. Hence $T=R$. If $R$ supersolvable then $G$ is solvable, a contradiction. Hence $H$ is weakly s-supplemented in $R$. But $R$ is the only supplement of $H$ in $R$, so $H=H \cap R$ is s-permutable in $R$ and hence $O_{p}(R) \neq 1$, which contradicts that $R$ is simple. This contradiction shows that (3) holds.

(4) $G$ is simple.

Let $N$ be a minimal normal subgroup of $G$. By (1) and (3), $N$ is a nonabelian $p d$-group. If $|P \cap N| \leq|D|$, then there is a subgroup $P_{1}$ of $P$ with $N \cap P<P_{1}$ and $\left|P_{1}\right|=p|D|$. Let $X=N P_{1}$. Then every maximal subgroup of the Sylow $p$-subgroup $P \cap X$ of $X$ is of order $|D|$. By Lemma 2.7 $X$ is $p$-nilpotent and so is $N$, a contradiction. If $|P \cap N|>|D|$, then the hypotheses hold on $N$. If $N<G$, then $N$ is $p$-solvable by the choice of $G$, a contradiction. Hence $N=G$ and $G$ is simple.

The final contradiction

If $p>2$, then $G$ is solvable and so is abelian, a contradiction. Hence $p=2$. Assume that $P$ is abelian and $H$ is a subgroup of $P$ of order $|D|$. If $H$ is weakly s-supplemented in $G$ and let $T$ be a supplement of $H$ in $G$ with $H \cap T \leq H_{s G}$. Then $T \neq G$ since $H$ could not s-permutable in $G$. Clearly, $P \cap T \neq 1$ since $D<P$. Hence $1 \neq(P \cap T)^{G}=(P \cap T)^{P T}=(P \cap T)^{T} \leq T<G$, which contradicts (4). Now consider that $P$ is nonabelian. Then every subgroup of order $|D|$ or $2|D|$ having no supersolvable supplement in $G$ is weakly s-supplemented in $G$. By Lemma 2.7 $D$ is not maximal in $P$ and so $2|D|<|P|$. If all subgroups of order $|D|$ (or of order $2|D|$ ) have supersolvable supplements in $G$, then all maximal subgroups of $P$ have supersolvable supplements in $G$. By Lemma 2.7 $G$ is $p$-nilpotent, a contradiction. Hence there is a subgroup $H_{1}$ of order $|D|$ and a subgroup $H_{2}$ of order $2|D|$ are weakly s-supplemented in $G$. But $G$ is simple, so both $H_{1}$ and $H_{2}$ are supplement in $G$. Hence there are subgroups $T_{1}$ and $T_{2}$ with $\left|G: T_{1}\right|=|D|$ and $\left|G: T_{2}\right|=2|D|$. In view Lemma 2.6 such a nonabelian simple group does not exist and our lemma holds.

Proof of Theorem 1.4 We first prove that $E$ satisfies Sylow tower property (see [11, p5]). In fact, by Lemma 2.1. it can be verified that the hypotheses still holds on $E$. If $E<G$ then $E \in \mathfrak{U}$ by induction and hence Esatisfies Sylow tower property in this case. Now assume that $E=G$. Let $p$ be the minimal prime divisor of $|G|$ and $P$ a Sylow $p$-subgroup of $G$. It follows from Lemma 3.4 that $G$ is $p$-solvable and the $p$-length of $G$ is 1 . Thus $G / O_{p^{\prime}}(G)$ is $p$-closed. By Lemma 2.1, every subgroup of $G / O_{p^{\prime}}(G)$ of order $|D|$ or $2|D|$ (when $P O_{p^{\prime}}(G) / O_{p^{\prime}}(G) \cong P$ is a nonabelian 2-group) having no supersolvable supplement in $G / O_{p^{\prime}}(G)$ is weakly s-supplemented in $G / O_{p^{\prime}}(G)$. If $\Phi(P) \neq$ $P^{\prime}$, then $P^{\prime} O_{p^{\prime}}(G) / O_{p^{\prime}}(G)<\Phi(P) O_{p^{\prime}}(G) / O_{p^{\prime}}(G) \leq \Phi\left(G / O_{p^{\prime}}(G)\right.$. It follows from Lemma 3.3 that $P O_{p^{\prime}}(G) / O_{p^{\prime}}(G) \subseteq Z_{\infty}^{\mathfrak{U}}\left(G / O_{p^{\prime}}(G)\right)$. Assume that $P^{\prime}=\Phi(P)$. If $|D| \leq|\Phi(P)|$ then $|D| \leq\left|P^{\prime}\right|$. Again by Lemma 3.3, it holds that $P O_{p^{\prime}}(G) / O_{p^{\prime}}(G) \subseteq Z_{\infty}^{\mathfrak{U}}\left(G / O_{p^{\prime}}(G)\right)$. Assume that $|D|>|\Phi(P)|$. 
Then (iii) holds on $P$ and so $\left(\iota\left(P / P^{\prime}\right), \iota\left(|D| /\left|P^{\prime}\right|\right)\right)=1$ or $(\iota(P), \iota(|P: D|))=1$. By Corollary 3.2 it can be verified that $P O_{p^{\prime}}(G) / P^{\prime} O_{p^{\prime}}(G) \subseteq Z_{\infty}^{\mathfrak{U}}\left(G / P^{\prime} O_{p^{\prime}}(G)\right)$, and it follows from Lemma 2.2 that $P O_{p^{\prime}}(G) / O_{p^{\prime}}(G) \subseteq Z_{\infty}^{\mathfrak{U}}\left(G / O_{p^{\prime}}(G)\right)$. Since $p$ is the minimal prime divisor of $G$, we have that $G / O_{p^{\prime}}(G)$ is $p$-nilpotent and hence $G$ is $p$-closed. Since the hypotheses still hold on $O_{p^{\prime}}(G)$, we have that $O_{p^{\prime}}(G)$ satisfies Sylow tower property, and consequently $G$ satisfies Sylow tower property.

Let $q$ be the maximal prime divisor of $|E|$ and $Q$ a Sylow $q$-subgroup of $E$. Then $Q$ char $E \unlhd G$ and as above argument, $Q \subseteq Z_{\infty}^{\mathfrak{U}}(G)$ since (i) or (ii) or (iii) holds on $Q$. We can also see that the hypotheses still holds on $G / Q$. Hence $G / Q \in \mathfrak{F}$ by induction on the order of $G$. Since $Q \subseteq Z_{\infty}^{\mathfrak{U}}(G)$ and $\mathfrak{U} \subseteq \mathfrak{F}$, we obtain that $G \in \mathfrak{F}$. Therefore the theorem holds.

\section{Remarks, examples and some corollaries}

1. If $D$ is minimal or maximal in $P$, then $(\iota(P), \iota(D))=1$ or $(\iota(P), \iota(|P: D|))=1$ and hence Theorems $\mathrm{A}$ and $\mathrm{B}$ in [7] are special cases of our results.

2. In Lemma 3.4 the minimality of $p$ is necessary. In fact, if $p$ is not the minimal prime divisor of the order of $|G|$, then we have the following counterexample.

Example 4.1 Let $A=Z_{3} \rtimes Z_{2} \cong S_{3}$, where $Z_{3}$ is a cyclic subgroup of order $3, Z_{2}$ a cyclic subgroup of order 2 and $S_{3}$ is the symmetric group of degree 3. Let $B=A$ 々 $Z_{3}$, the regular wreath product of $A$ by $Z_{3}$. Put $G=O^{2}(B)=\langle x \mid o(x)=3\rangle$. Then $G \cong\left(Z_{3} \times Z_{3} \times Z_{3}\right) \rtimes A_{4}$, where $A_{4}$ is the alternative group of degree 4. Let $P$ be a Sylow 3-subgroup of $G$. It can be proved that for any maximal subgroup $H$ of $P, H$ is complemented in $G$. So every maximal subgroup of $P$ is weakly s-supplemented in $G$. But the p-length of $G$ is not 1 , where $p=3$.

3. Clearly, if a subgroup $H$ is normal, s-permutable or c-normal in $G$, then $H$ is weakly-supplement in $G$. Hence one can find the following special cases of Theorem 1.4 in the literature.

Corollary 4.2 ( [12]) Let $G$ be a group of odd order. If all subgroups of $G$ of prime order are normal in $G$, then $G$ is supersolvable.

Corollary 4.3 ( [13]) If the maximal subgroups of the Sylow subgroups of $G$ are normal in $G$, then $G$ is supersolvable.

Corollary 4.4 ( [14]) If all subgroups of $G$ of prime order or order 4 are c-normal in $G$, then $G$ is supersolvable.

Corollary 4.5 ( 14]) If the maximal subgroups of the Sylow subgroups of $G$ are c-normal in $G$, then $G$ is supersolvable.

Corollary 4.6 ( [15]) If the maximal subgroups of the Sylow subgroups of $G$ not having supersolvable supplement in $G$ are normal in $G$, then $G$ is supersolvable.

Corollary 4.7 ( [16]) If the maximal subgroups of the Sylow subgroups of $G$ not having supersolvable supplement in $G$ are c-normal in $G$, then $G$ is supersolvable. 
Corollary 4.8 ( [17]) Let $\mathfrak{F}$ be a saturated formation containing $\mathfrak{U}$. If all minimal subgroups and all cyclic subgroups with order 4 of $G^{\mathfrak{F}}$ are c-normal in $G$, then $G \in \mathfrak{F}$.

Corollary 4.9 ( [18]) Let $\mathfrak{F}$ be a saturated formation containing $\mathfrak{U}$ and $G$ a group with normal subgroup $E$ such that $G / E \in \mathfrak{F}$. Assume that a Sylow 2-subgroup of $G$ is abelian. If all minimal subgroups of $E$ are permutable in $G$, then $G \in \mathfrak{F}$.

Corollary 4.10 ( [19]) Let $G$ be a solvable group. If all maximal subgroups of the Sylow subgroups of $F(E)$ are normal in $G$, then $G$ is supersolvable.

Corollary 4.11 ( [18]) Let $\mathfrak{F}$ be a saturated formation containing $\mathfrak{U}$ and $G$ a group with a solvable normal subgroup $E$ such that $G / E \in \mathfrak{F}$. If all minimal subgroups and all cyclic subgroups with order 4 of $E$ are weakly s-permutable in $G$, then $G \in \mathfrak{F}$.

\section{References}

[1] K. Doerk and T. Hawkes. Finite soluble groups. Walter de Gruyter \& Co., Berlin, 1992.

[2] B. Huppert. Endliche Gruppen. I. Springer-Verlag, Berlin, 1967.

[3] W. Guo. The theory of classes of groups. Kluwer Academic Publishers Group, Dordrecht, 2000.

[4] O. Ore. Contributions to the theory of groups of finite order. Duke Math. J., 5(2):431-460, 1939.

[5] O. Kegel. Sylow-Gruppen und Subnormalteiler endlicher Gruppen. Math. Z., 78:205-221, 1962.

[6] A. Skiba. On weakly s-permutable subgroups of finite groups. J. Algebra, 315(1):192-209, 2007.

[7] W. Guo, F. Xie, and B. Li. Some open questions in the theory of generalized permutable subgroups. Sci. China Ser. A, 52(10):2132-2144, 2009.

[8] B. Li and W. Guo. On some open problems related to $X$-permutability of subgroups. Comm. Algebra, 39:757-771, 2011.

[9] R. Guralnick. Subgroups of prime power index in a simple group. J. Algebra, 81(2):304-311, 1983.

[10] B. Li. On П-property and П-normality of subgroups of finite groups. J. Algebra, 334:321-337, 2011.

[11] M. Weinstein(ed.). Between nilpotent and solvable. Polygonal Publ. House, Washington, N. J., 1982.

[12] J. Buckley. Finite groups whose minimal subgroups are normal. Math. Z., 116:15-17, 1970.

[13] S. Srinivasan. Two sufficient conditions for supersolvability of finite groups. Israel J. Math., 35(3):210$214,1980$.

[14] Y. Wang. c-normality of groups and its properties. J. Algebra, 180(3):954-965, 1996.

[15] W. Guo, K. P. Shum, and A. Skiba. G-covering subgroup systems for the classes of supersoluble and nilpotent groups. Israel J. Math., 138:125-138, 2003.

[16] A. Ahmad. Finite group with given c-permutable subgroups. Algebra Discrete Math., (2):9-16, 2004. 
[17] A. Ballester-Bolinches and Y. Wang. Finite groups with some $C$-normal minimal subgroups. J. Pure Appl. Algebra, 153(2):121-127, 2000.

[18] A. Ballester-Bolinches and M. C. Pedraza-Aguilera. On minimal subgroups of finite groups. Acta Math. Hungar., 73(4):335-342, 1996.

[19] M. Ramadan. Influence of normality on maximal subgroups of Sylow subgroups of a finite group. Acta Math. Hungar., 59(1-2):107-110, 1992. 